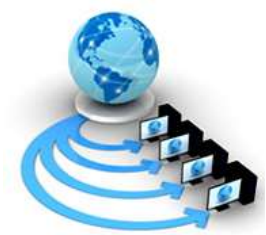

Volume 9, No. 6, November-December 2018

ISSN No. 0976-5697

International Journal of Advanced Research in Computer Science

RESEARCH PAPER

Available Online at www.ijarcs.info

\title{
SIMITS2: SIMULATION FRAMEWORK FOR VEHICULAR CLOUD COMPUTING
}

\author{
Nayana Hegde \\ Dept.of Electronics and Communication \\ Sri Krishna Institute of Technology Bengaluru, \\ India
}

\author{
Sunilkumar S. Manvi \\ Dept. of CIT \\ REVA University Bengaluru, \\ India
}

\begin{abstract}
Vehicular Cloud is a hybrid technology comprising of VANET, cloud computing and wireless communication. VCC facilitates convenient, on demand access to network, storage area, applications and services on pay as you use basis. VCC is formed by pooling the resources from moving vehicles. Due to the prohibitive cost of deploying and implementing such as system in a real world, most research work in VCC rely on simulations for evaluation purpose. The key concept for VCC simulations is a real world vehicular mobility model. In this paper we present a tool SIMITS2 that allows users to easily generate real world mobility models for VCC simulations. The simulation results are obtained when vehicles are moving according to a real world scenario.
\end{abstract}

Keywords: VCC, Simulator, Cloud computing, VANET.

\section{INTRODUTION}

Recently VCC has drawn noteworthy attention from researchers and academicians. VCC is a network of smart vehicles, where vehicles communicate with vehicles (V2V) and infrastructures (V2I) in real time, to realize the vision of intelligent Transportation Systems (ITS) [1]. A smart vehicle consists of sensing, computing, storing and communicating resources. The smart vehicle in VCC communicates with internet to get required information while travelling. It is costly to communicate with infrastructure for every vehicle. Moreover, many vehicles may need information of their local vicinity only. So neighboring vehicles can pool their unutilized resources and cloud is formed [2][5].

The ability to collect sensor information which is of local relevance is biggest advantage of vehicular cloud. VCC is gaining popularity due to wide range of applications. Applications are mainly safety related, entertainment and military related [3][4].

It is very important to test and assess protocol implementations in a real world environment. Simulations are commonly used as a first step in the protocol development for research [6]. Several communicationsnetwork simulator already exist to provide a platform for testing and evaluating network protocols. We highlight the simulators that support the new IEEE 802.11p standard for data exchange in high speed V2V and V2I scenarios. Next section we list the simulators with their unique features.

Objectives of proposed paper are as follows

1. List the network simulators and discuss their unique features

2. Explain SIMITS2 with its features

3. Evaluate performance of VCC using SIMITS2.

The paper has been divided into five sections. The first section introduces the readers to the basic of VCC and our contribution. The second section gives list of network simulator. Third section explains SIMITS2 simulator. Forth section is evaluation of VCC. Last is conclusion.

\section{NETWORK SIMULATORS}

The basic function of a network simulator is to analyze the performance of network protocols under different network topologies and settings. Although there are many network simulators available, we are particularly interested in network simulators that are suitable to use with the vehicular network simulations. We highlight the simulators that support the new IEEE $802.11 \mathrm{p}$ standard for data exchange in high speed V2V and V2I scenarios without involving association and authentication mechanism of vanilla 802.11 standards. Following is the list of vehicular network simulators with their important features.

\section{- NS-2 (Network Simulator 2)}

Network Simulator (Version 2), widely known as NS2, is simply an event driven simulation tool that has proved useful in studying the dynamic nature of communication networks [7].

\section{- (Network Simulator 3)}

NS-3 is a discrete-event network simulator for Internet systems, targeted primarily for research and educational use. NS-3 is free software, licensed under the GNU GPLV2 license, and is publicly available for research, development.

\section{- OPNET}

OPNET stood for Optimized Network Engineering Tools. It is a software tool for computer network modelling and simulation. It is good for application and network performance management capabilities.

\section{- Mininet}


Mininet is a network emulator which creates a network of virtual hosts, switches, controllers, and links. IT hosts run standard Linux network software, and its switches support open flow for highly flexible custom routing and Software-Defined Networking.

\section{- $\quad$ OMNet +}

It is a modular, component-based $\mathrm{C}++$ simulation library and framework, primarily for building network simulators. OMNeT++ can be used for free for noncommercial simulations like at academic institutions and for teaching.

\section{- JiST}

JiST is a high-performance discrete event simulation engine that runs over a standard Java virtual machine. It is a prototype of a new general-purpose approach to building discrete event simulators, called virtual machine-based simulation that unifies the traditional systems and language-based simulator designs. The resulting simulation platform is surprisingly efficient. It out-performs existing highly optimized simulation runtimes both in time and memory consumption [8].

\section{- MobiReal}

MobiREAL is a novel network simulator for ubiquitous society with mobile devices. It can simulate realistic mobility of humans and automobiles, and enables to change their behaviour depending on a given application context. Therefore, we can get more detailed performance evaluation of network applications, routing protocols, infrastructure and so on which existing simulators cannot evaluate well.

\section{- MobiSim}

MobiSim is a mobility management application, composed of many modules, using a pluggable architecture and a form generator in order to help researchers in the field of MANET (Mobile Ad-hoc NETworks) to deal with mobility in these networks. User can create "scenarios", simulate them in graphical or batch modes, simulate the traces, evaluate measures on the traces, create Excel diagrams by means of the evaluations, and finally test mobility model recognition algorithms on the traces. It supports plain text, NS format, and $\mathrm{xml}$ files for traces and contains many mobility models, which can be mixed together and configured graphically. All of these can be extended with an XML configuration file without any need to create additional GUI and recompiling thanks to the form generator and Parameter framework.

\section{- NetSim}

NetSim is a discrete event simulator covering a broad range of wired, wireless, mobile and sensor networks. It comes with a simple and user friendly GUI which features drag and drop functionality for devices, links, application etc.

\section{SIMITS2}

SIMITS2 is communication simulator in the field of ITS. It is able to simulate and to measure the performance of different medium access control mechanisms (MAC). The simulation experiments can be performed considering a convoy of autonomous vehicles which follow a trajectory on a limited area. To guarantee the convoy stability, certain messages must be interchanged among the vehicles members of the convoy. The software allows modifying several parameters as the number of vehicles, trajectory, environment, etc It also presents the evolution and the results of the experiment in screen and in *.csv files. This software has been written in C\#. Source files are .cs files in the simulator software. Compiled files are .exe files or .dll files. The complete project source files are bundled to .sln file. It is called solution file. Figure 1 depicts details of configuration of simulator files.

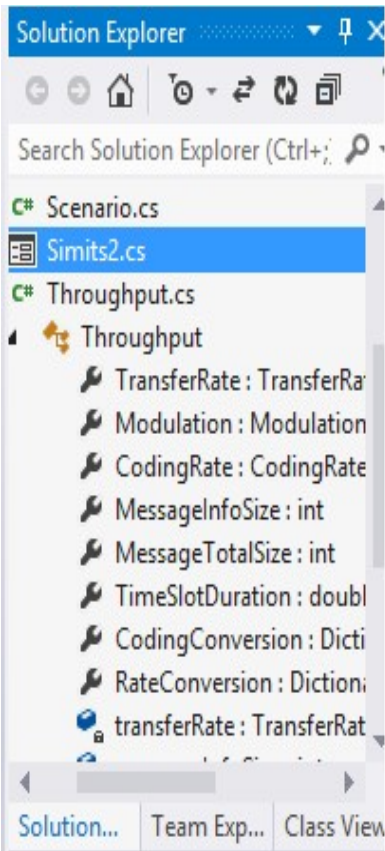

Figure 1. Configuration of Simulator source files

SIMITS2 is an open-source simulation platform, aiming at a realistic evaluation of protocols for large scale vehicular networks. SimITS2 aims to simulate properly both road traffic conditions and wireless communications characteristics and at the same time to ensure the real time exchange of simulation data between them. Figure 2 shows the architecture of the simITS 2 simulator. It consists of four components. The components are: GUI, interface, core and results. GUI of simulator is shown is shown in figure 3. It is user interface, which helps user to input all values for simulation. Controller which consists of event handler programs of the simulator. Core consists of the programs which are required to handle the events of simulator. When the simulator evaluation is started, the results of the simulation can be seen in graphical results window, as shown in figure 4. After simulation is completed, the output of the simulation is written into .csv file. These output files 
are named with timestamp of the simulation. Format of the simulation file is shown in figure 5 .

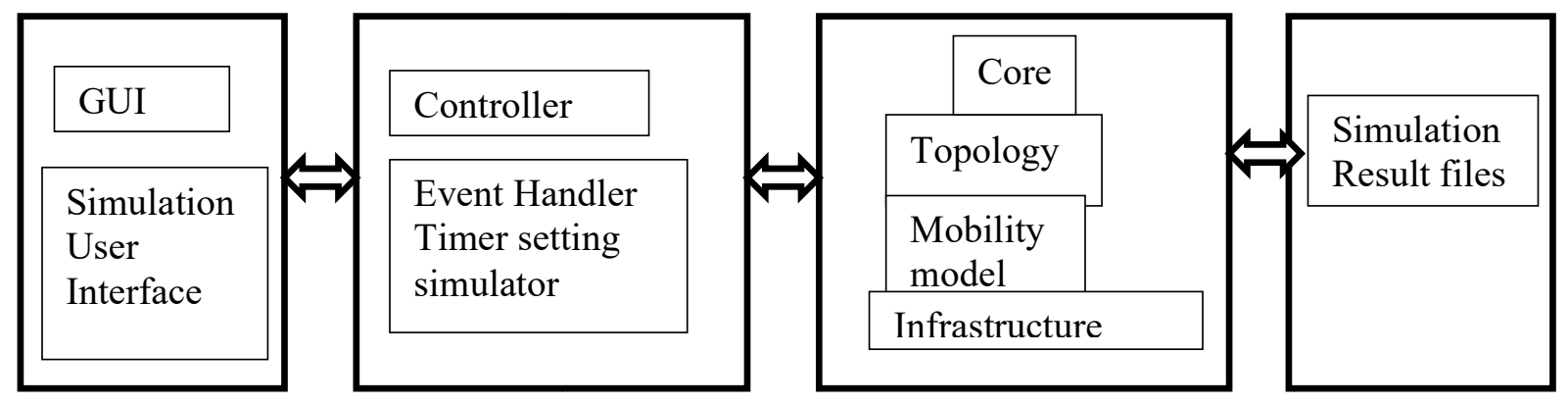

Figure 2.Architecture of the SimITS2 platform

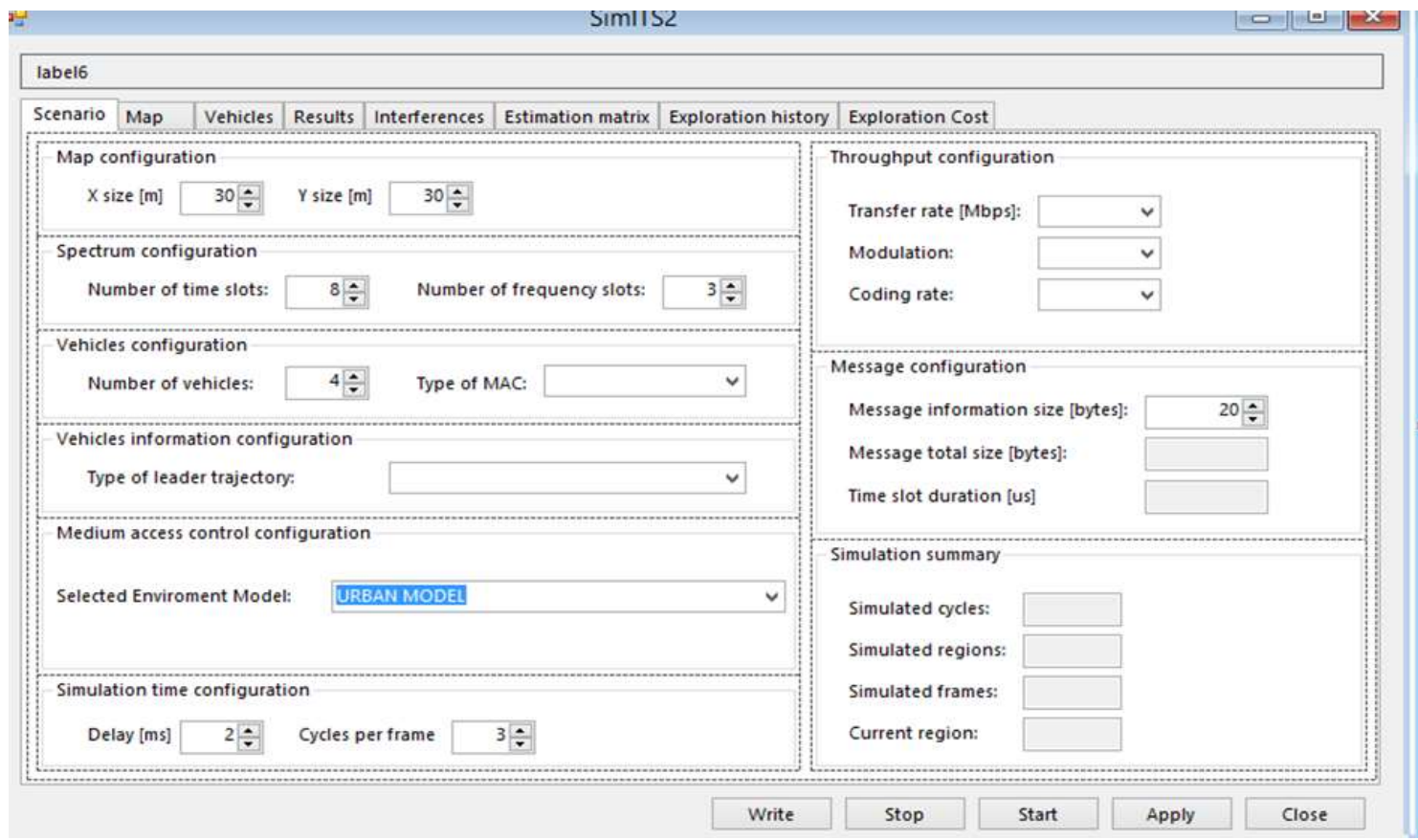

Figure3. GUI of SimITS2simulator for input scenario

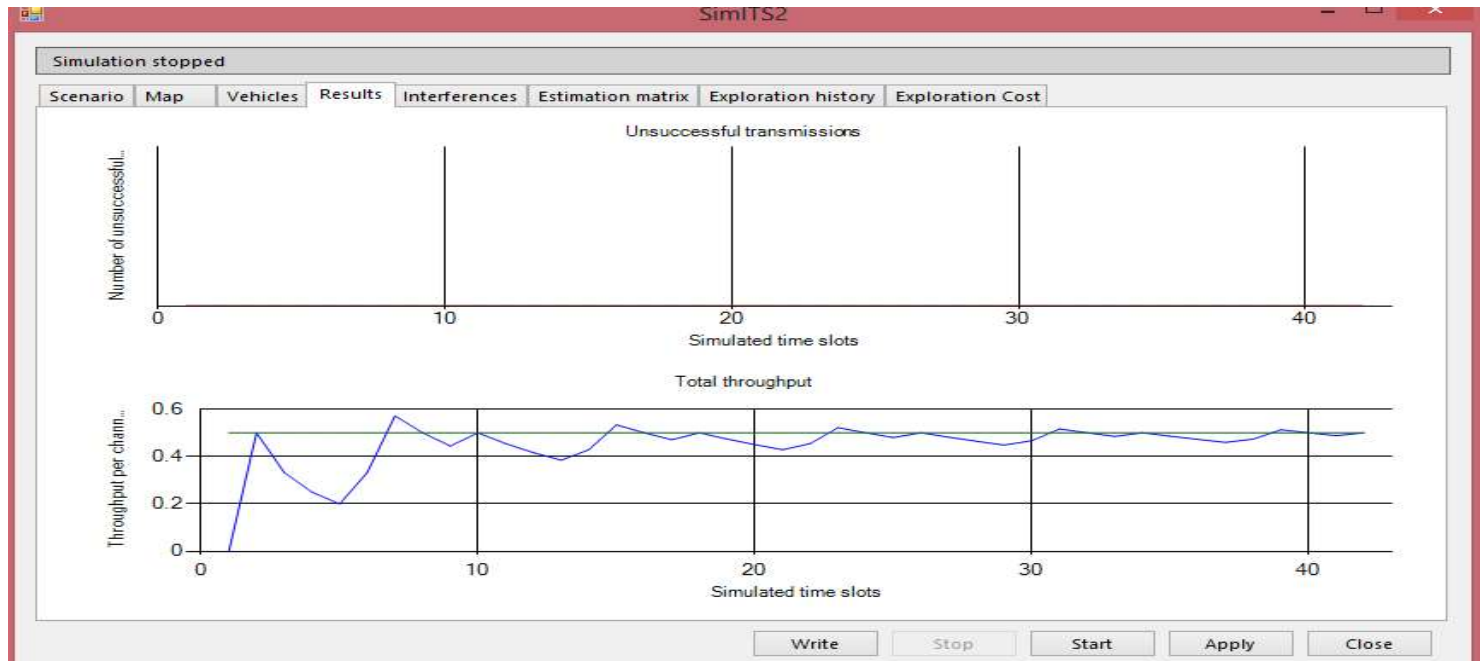


Figure 4. Results of SimITS2simulator

\begin{tabular}{|c|c|c|c|}
\hline MAC & Time slots & Frequency slots & Number of users \\
\hline NCCMA & 8 & 3 & 4 \\
\hline Transfer rate [Mbps] & Modulation & Coding Rate & Maximum throughput [Mbps] \\
\hline 3 & BPSK & 0.5 & 0.5 \\
\hline Information size [bytes] & Total size [bytes] & Time slot duration [us] & \\
\hline 20 & 40 & 106.6666667 & \\
\hline Vehicle id & Region used & Frame & TxSuccess \\
\hline 1 & 16 & 0 & 2 \\
\hline 1 & 16 & 1 & 6 \\
\hline 1 & 16 & 2 & 10 \\
\hline 1 & 16 & 3 & 14 \\
\hline 1 & 16 & 4 & 18 \\
\hline 2 & 4 & 0 & 1 \\
\hline 2 & 4 & 1 & 5 \\
\hline 2 & 4 & 2 & 9 \\
\hline 2 & 4 & 3 & 13 \\
\hline 2 & 4 & 4 & 17 \\
\hline 2 & 4 & 5 & 21 \\
\hline
\end{tabular}

Figure 5. Output file format

\section{EVALUATION}

This section depicts the results which are obtained by running the SIMITS2, an open-source simulator for dynamic vehicular cloud. It performs vehicle to infrastructure communication using IEEE $802.11 \mathrm{p}$ standard. The simulation includes urban and highway scenario and vehicles moving in single lane one way road. Distance between each vehicle is less than 30 meters. Simulation parameters are as follows: time slot duration 160 Micro second, number of vehicles $10 / 20$, information size $20 / 30$ bytes and data rate of $6 \mathrm{Mbps}$. Figure 6 shows the graph obtained throughput per channel for 20 users in urban scenario. Simulation is carried for $65 \mathrm{secs}$ of time slot. Figure 7 shows the number of transaction with time for 20 vehicle users.

\section{Throughput per channel}

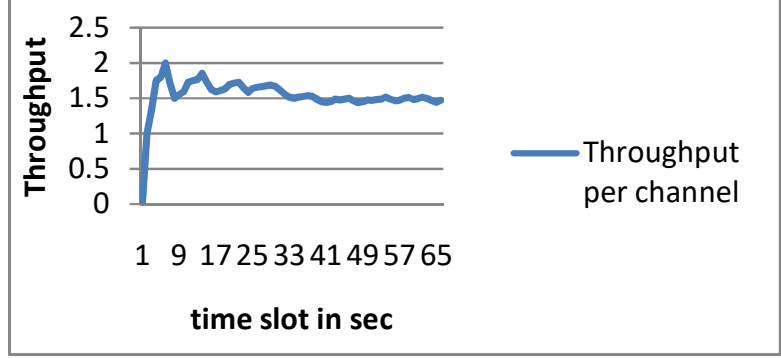

Figure 6.Throughput per channel for 20 users

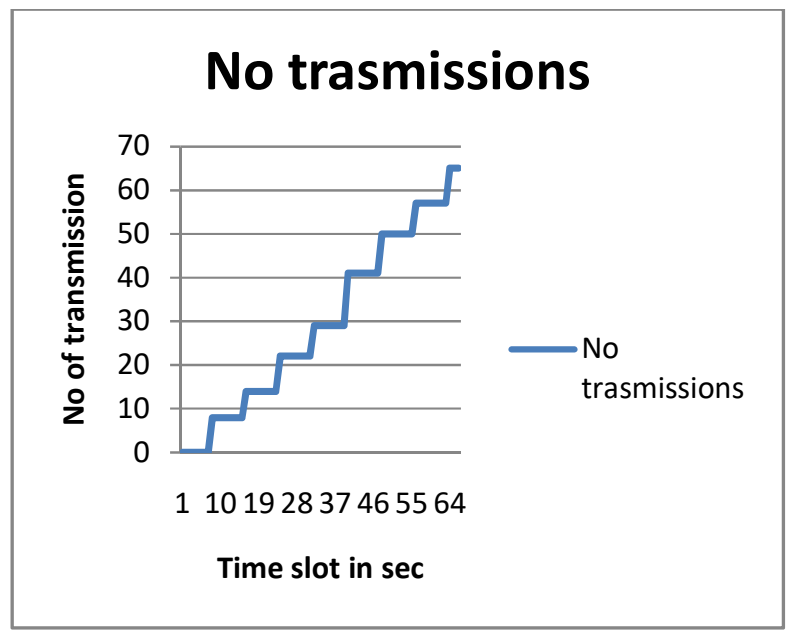

Figure 7.No transmissions for 20 users

\section{5 .CONCLUSION}

In this paper, we describe the simulation of vehicular cloud network using SIMITS2 tool on windowsoperating system. It is based on an open source .net framework. SIMITS2 allows user to quickly and easily generate real world 
mobility models for vehicular cloud simulations. Next step is to use this tool to understand the effect of level of details in context of VCC simulation. The movements of vehicles are based on static configurations and it can be of various patterns in urban, rural or highway environment. The mobility model is decided depending on the requirement. Simulation results can documented easily with the helpof output file generated by the simulator.

\section{REFERENCES}

[1]Sunilkumar S. Manvi, Nayana Hegde,’Key Management Authentication and Non Repudiation for Information Transaction in Vehicular Cloud Environment “, IEEE International Conference on Cloud Computing in Emerging Markets (CCEM), 2016

[2] K. Z. Ghafoor, K. A. Bakar, M. A. Mohammed, and J. Lloret, "Vehicular cloud computing: Trends and challenges," Igi global chapter, pp. 1-7, 2013.

[3]Sunilkumar S. Manvi, Nayana Hegde, "Emerging Applications in Vehicular Cloud Computing", International Conference on Computer Communication and Informatics, Coimbatore, Tamil Nadu, 2017
[4] M. Whaiduzzaman, M. Sookhak, A. Gani, and B. Rajkumar, "A survey on vehicular cloud computing," Journal of Network and Computer Applications, vol. 40, no. 1, pp. 325-344, 2014.

[5]M. Eltoweissy, S. Olariu, and M. Younis, "Towards autonomous vehicular clouds," International Conference on Ad Hoc Networks, pp. 1-16, 2010.ad hoc networks, IEEE Transactions on Vehicular Technology 66 (7) (2017) 6336-6348.

[6]Vaishali D. Khairnar, S.N.Pradhan, "Mobility Models for Vehicular Ad-hoc Network Simulation",International Journal of Computer Applications,Volume 11- No.4, December 2010, 8-12

[7] Nayana Hegde,Sunilkumar.S.Manvi,"Simulation of Wireless Sensor Network Security Model Using NS2", International Journal of Latest Trends in Engineering and Technology, Vol. 4 Issue 1 May 2014 pp.113-119.

[8]R. Barr, Z. J. Haas, R. van Renesse, Jist/swans, Wireless Networks Laboratory, Cornell University. http://jist. ece. ornell. du

[9] X. Zhou, J. Taylor, Dtalite: A queue-based mesoscopic traffic simulator for fast model evaluation and calibration, Cogent Engineering 1 (2014)1-19. 